\title{
ANÁLISIS EXPLORATORIO DEL DESPERDICIO DE ALIMENTOS EN PLATO EN COMEDORES ESCOLARES EN ESPAÑA
}

\author{
EXPLORATORY ANALYSIS OF FOOD WASTE AT PLATE IN SCHOOL CANTEENS IN \\ SPAIN
}

\author{
María Bustamente ${ }^{1}$, Ana Afonso², \\ Ignacio De los Ríos ${ }^{1 *}$
}

\footnotetext{
1* Cátedra Bancos de Alimentos de la Universidad Politécnica de Madrid, Calle Ramiro de Maeztu, 7, 28040 Madrid, España.

2 Escuela de Organización Industrial, Av. Gregorio del Amo 6, 28040, Madrid, España
}

*Autor para correspondencia: ignacio.delosrios@upm.es

\begin{abstract}
Resumen
La investigación se enmarca en una relación Universidad-Sociedad civil, que se articula en la Cátedra Bancos de Alimentos UPM-FESBAL, como un medio para establecer una colaboración estratégica a largo plazo, con el fin de "Trabajar con la gente", para conseguir un consumo racional y solidario. Comprender mejor el proceso de generación de desperdicios en los comedores escolares representa una oportunidad clara para la aplicación de medidas frente al desperdicio alimentario, debido a que la infancia es el mejor momento para crear conciencia sobre la importancia de abordar este problema. El desperdicio en plato en comedores escolares es causado por la poca receptividad de los alumnos frente al menú, lo que se ve afectado por factores propios de cada comensal y por factores contextuales. La presente investigación tiene como objetivo aproximar la cantidad de desperdicio en plato en comedores escolares en España e identificar posibles factores condicionantes a nivel contextual, a partir del análisis de 118 comedores escolares en 14 comunidades autónomas. Los resultados muestran que, a nivel territorial, existen diferencias significativas entre los desperdicios en costa e interior. A nivel de centro educativo, sucede lo mismo entre los desperdicios medidos antes y después de actividades de sensibilización. Finalmente, los resultados muestran que el desperdicio por tipo de menú difiere según la región en la que se ubica el centro educativo, lo que da indicios de la relación entre la región y el grado de aceptación o preferencias por menús.
\end{abstract}

Palabras claves: Desperdicio alimentario; desperdicio en plato; comedores escolares; factores contextuales; consumo racional; universidad-sociedad civil. 


\begin{abstract}
The research is part of a University-Civil Society relationship, which is framed on UPM-FESBAL Food Banks Chair, as a means to establish a long-term strategic collaboration, in order to "Work with people", to achieve a rational and supportive consumption. A better understanding of food waste generation in school canteens represents a clear opportunity for the application of measures against it, because childhood is considered the best time to raise awareness about this problem and their impacts. Plate waste in school canteens depends of the student's receptivity for each menu, which is affected by individual and contextual factors. The aim of this research is to approximate the amount of plate waste in school canteens in Spain and identified possible drivers at contextual level, based on the analysis of 118 school canteens in 14 autonomous communities. The results show that, at a territorial level, there are significant differences between plate waste on the coast and interior. At school level, the same thing happens between the waste measured before and after the awareness campaign. Finally, the results show that the plate waste per type of menu differs according to the region where the school is located, which suggest that the acceptance or student's preferences for menus are related with the region where the school is located.

Keywords: Food waste; plate waste; school canteens; contextual factors; university- society; rational consumption.

Forma sugerida de citar: Bustamanet, M., Afonso, A. y De los Ríos, I. 2018. Análisis exploratorio del desperdicio de alimentos en plato en Comedores Escolares en España. La Granja: Revista de Ciencias de la Vida. Vol. 28(2):20-42. http://doi.org/10.17163/lgr.n28.2018.02.
\end{abstract}




\section{Introducción}

En el 2011, la Organización de las Naciones Unidas para la Agricultura y la Alimentación (FAO, por sus siglas en inglés) señaló que para suplir las necesidades alimentarias de una población que se estimó pasaría de 7000 a 9000 millones de personas para el 2050, sería necesario incrementar la producción en un $70 \%$. En contraposición con el problema de inseguridad alimentaria, FAO (2011) señaló que aproximadamente un tercio de los alimentos producidos en el mundo se perdían o desperdiciaban. Desde ese año, el problema de desperdicio y pérdida de alimentos ha tomado relevancia en la lucha contra la pobreza, el hambre, el cambio climático y el uso inadecuado de recursos naturales.

Lipinski y Lomax (2013) estiman que, si se redujeran a la mitad las pérdidas y desperdicios de alimentos, la brecha para suplir las necesidades alimentarias pronosticadas por FAO para el 2050 se reduciría a la mitad. Además, los mismos autores señalan que la pérdida y desperdicio de alimentos equivale a una inversión desperdiciada que reduce el bienestar económico de los actores de la cadena de valor de los alimentos.

En relación con los impactos ambientales, FAO (2014) señala las pérdidas de los recursos naturales empleados para producir los alimentos que son desperdiciados o impactados en la producción de los mismos; además enfatiza en la emisión de $\mathrm{CO}_{2}$-eq por el desperdicio de alimentos en el mundo a lo largo de la cadena de suministro.

El estudio FUSIONS de la Comisión Europea (Stenmarck et al., 2016) estima el desperdicio de alimentos para el 2012 en 88 millones de toneladas, lo que equivale a 173 kilogramos de desperdicio anual por persona en la Unión Europea (UE-28). Stenmarck et al. (2016) señalan también que la mayor parte de los desperdicios en la Unión Europea se registran en las etapas finales de la cadena de suministro de alimentos-53\% en hogares, $12 \%$ en restauración colectiva y $5 \%$ en comercialización-lo que se traduce en mayores impactos ambientales y una mayor cantidad de dinero despilfarrado (FAO, 2011). Por estas razones, la prevención del desperdicio cobra mayor importancia al final de la cadena (Eriksson et al., 2017).

Si bien el sector de hogares representa una fuente significativa de desperdicio a nivel del consumidor, las instituciones que brindan servicios de alimentación-escuelas, prisiones y hospitales-son también una fuente importante de desperdicios en países desarrollados (Cordingley, Reeve y Stephenson, 2011). Los comedores escolares, por su condición de servir alimentos a estudiantes concentrados en un lugar, representan una oportunidad ideal para aplicar medidas orientadas a prevenir y/o reducir los desperdicios de alimentos (Wilkie, Graunke y Cornejo, 2015). Esto se ve reforzado por una sociedad que demanda a las instituciones públicas y privadas, cada vez más, el desarrollo de iniciativas que contribuyen a lograr un desarrollo sostenible (Derqui y Agustín, 2016).

Para reducir el desperdicio de alimentos es necesario entender el problema a resolver, por esta razón es crítico desarrollar una cuantificación detallada del desperdicio (Eriksson et al., 2017; World Resources Institute, 2016). En los últimos años se han realizado varios estudios que analizan el desperdicio en los comedores escolares u otros centros de restauración colectiva, y la mayoría se han centrado en analizar los desperdicios en plato motivados por preocupaciones nutricionales (Engström y Carlsson-Kanyama, 2004; Wilkie, Graunke y Cornejo, 2015). Engström y Carlsson-Kanyama indican que los estudios existentes presentan tasas de generación de residuos altamente variables entre sí, debido posiblemente a que las mediciones se suelen hacer en períodos cortos de tiempo (Eriksson et al., 2017), o a que al presentar el resultado de la medición de los desperdicios, no se evidencia el efecto de factores condicionantes sobre la variabilidad de los datos (Secondi, Principato y Laureti, 2015).

En España, el Ministerio de Agricultura y Pesca, Alimentación y Medio Ambiente (Ministerio de Agricultura y Pesca, Alimentación y Medio Ambiente, 2017) como parte de su estrategia Más alimento, menos desperdicio contempla el desarrollo de estudios que permitan una mayor comprensión del fenómeno de generación de desperdicios alimentarios. Para los comedores escolares, se realizó un estudio piloto en cuatro centros educativos, presentando alta variabilidad en los resultados.

Por lo antes mencionado, y conociendo la importancia del desperdicio alimentario en comedores escolares, se considera necesario realizar estudios de desperdicios alimentarios, contemplando los posibles factores condicionantes de este, de manera que sea posible explicar la alta variabilidad de los datos. Por otro lado, es necesario realizar investigaciones que contemplen muestras de mayor tamaño, durante un mayor período de tiempo y cuyos objetivos no 
se centren únicamente en aspectos nutricionales, si no que contemplen los impactos del desperdicio a otros niveles.

En la presente investigación se realiza un análisis exploratorio de la cantidad de desperdicio en plato en comedores escolares en España, y su relación con factores contextuales-propios del territorio y del centro educativo-con el objetivo de identificar posibles condicionantes de la heterogeneidad en el desperdicio en plato en comedores escolares en España. La investigación se enmarca en la Cátedra Banco de Alimentos-UPM (CBA), como una nueva forma de construir relaciones UniversidadEmpresa-Sociedad de cara al consumo racional (De los Ríos et al., 2015). La idea surge desde la Federación Española de Bancos de Alimentos (FESBAL) que busca al Grupo de Investigación en Planificación y Gestión Sostenible del Desarrollo Rural Local (GESPLAN) de la Universidad Politécnica de Madrid (UPM). Desde la actividad de FESBAL, España ocupa el primer lugar en Europa en actividad solidaria, con 55 Bancos de Alimentos, sobre un total de 257 Bancos en Europa (FAO, 2014). La actividad de FESBAL se integra en la FEBA (Fédération Europenne des Banques Alimentaires) que aglutina 257 Bancos que operan en 22 países de Europa (FESBAL, 2017).

\section{Revisión de estudios previos}

Partiendo de la importancia de profundizar en el análisis de desperdicios alimentarios, en este apartado se presenta una revisión de las principales consideraciones para el desarrollo de inventarios y análisis de desperdicios alimentarios. Además, se resumen los resultados de estudios que cuantifican el desperdicio en plato y exploran el fenómeno de generación de este desperdicio a partir de la identificación y/o revisión de factores condicionantes.

\subsection{Consideraciones al realizar inventarios y análisis de desperdicios alimentarios}

Para estudiar los desperdicios y/o pérdida de alimentos a lo largo de la cadena, es necesario abordar tres definiciones clave empleadas en la literatura sobre este tema: pérdida, desperdicio y excedente. La pérdida y el desperdicio de alimentos se diferencian de acuerdo con la etapa en la que se producen; así tenemos que la pérdida o deterioro ocurre en las etapas de producción, post-cosecha y procesamiento, y está relacionada con la necesidad de invertir en infraestructura y tecnología, mientras que en las etapas finales-comercialización y consumo final-se emplea el término desperdicio, y se relaciona principalmente con aspectos de comportamiento (Parfitt, Barthel y Macnaughton, 2010); otros autores distinguen ambos términos por la naturaleza de sus causas, atribuyendo causas de comportamiento a los desperdicios (HLPE, 2014). Por otro lado, el excedente se define como todos los alimentos que se producen más allá de nuestras necesidades nutricionales, mientras que el desperdicio es producto de este excedente (Papargyropoulou et al., 2014).

Es importante también, para proponer una adecuada estrategia de prevención de desperdicios, distinguir entre desperdicios evitables y no evitables (Papargyropoulou et al., 2014). El desperdicio evitable es todo lo que en algún momento fue comestible, y que en el momento de ser desechado podría o no seguir siéndolo (Derqui y Fernández, 2017).

Con el fin de comprender el proceso por el cual se generan pérdidas y desperdicio de alimentos, se vienen desarrollado protocolos, estrategias y metodologías de medición tanto a nivel global como por países. A nivel global, las instituciones socias del Protocolo de Pérdida y Desperdicio de Alimentos desarrollaron el Estándar Global de Medición de Pérdida y Desperdicio de Alimentos (FLW Standard), con el objetivo de dar a conocer a los gobiernos e instituciones los requisitos para realizar una buena medición, y brindarles orientación en el proceso (World Resources Institute, 2016).

Independientemente del objetivo de la medición, el World Resources Institute (2016) considera importante tener información clara sobre cuatro elementos: Plazos, límites, alcance y destino del desperdicio. En alcance se debe incluir información sobre el tipo de desperdicio analizado, las causas de su generación y una cuantificación estimada.

En España, el Ministerio de Agricultura y Pesca, Alimentación y Medio Ambiente (2017) contempla, como parte de su estrategia Más alimento, menos desperdicio, el desarrollo de estudios que contribuyan a responder cuánto, cómo, dónde y porqué del desperdicio alimentario en distintos sectores. En el piloto en comedores escolares, se aplicó una metodología que parte de la compresión de tres ámbitos: el marco regulatorio, los procesos de elaboración de los alimentos y las condicionantes o causas del desperdicio (Derqui y Agustín, 2016). Este últi- 
mo ámbito es el que delimita el alcance del estudio, ya que determina el tipo de desperdicio a analizar y sus causas, de acuerdo con el momento en el que es generado (Ver Tabla 1).

El desperdicio en plato es un término empleado por los investigadores para referirse a la cantidad de comida servida en el plato de los comensales, que finalmente no es consumido. Usualmente se mide con el objetivo de evaluar el grado de aceptación de los alimentos por los comensales, medir la eficiencia del servicio de alimentación y/o evaluar la ingesta nutricional (Derqui y Fernández, 2017; Martins et al., 2014).
Según Buzby, Guthrie et al. (2002) el método más adecuado para medir desperdicio en plato es el pesado por tipo de alimentos, considerando los pesos iniciales de los platos y el peso de los desperdicios por cada comensal. Para simplificar y acelerar la colección de datos se suelen emplear métodos agregados, en los que se realiza el pesado total de los desperdicios y se obtiene el promedio por cada comensal. Se pueden dividir estos métodos en agregados selectivos y no selectivos; los primeros requieren diferenciar el desperdicio por tipo de alimentos, a diferencia de los no selectivos que realizan un pesado total de los desperdicios (Martins et al., 2014).

Tabla 1. Causas del desperdicio alimentario según el momento de su generación (Derqui y Agustín, 2016).

\begin{tabular}{cccc}
\hline & \multicolumn{3}{c}{ Momento de generación del desperdicio } \\
\cline { 2 - 4 } Causas & Antes de servir los alimentos & Luego de servir los alimentos \\
\hline \multirow{3}{*}{ Resultado } & $\begin{array}{c}\text { Procesos de } \\
\text { preparación, } \\
\text { almacenamiento } \\
\text { y manipulación } \\
\text { de alimentos. }\end{array}$ & Receptividad del alumno \\
& yesperdicios por sobreproducción, & $\begin{array}{c}\text { Desperdicios en cocina } \\
\text { y en línea (cocinado } \\
\text { no servido). }\end{array}$ & Desperdicios en plato \\
\hline
\end{tabular}

2.2 Estudios previos de cuantificación y condicionantes del desperdicio alimentario

Diversos estudios han cuantificado el desperdicio de alimentos en plato en el sector de restauración colectiva. Eriksson et al. (2017) obtuvieron en su estudio 24,75 gramos de desperdicio promedio en plato por porción servida en el sector de restauración en una municipalidad sueca, y Engström y Carlsson-Kanyama (2004) obtuvieron también en Suecia entre 27 y 33 gramos $(9-11 \%$ de la ración servida) -principalmente patatas, arroz y pasta-en un análisis exploratorio en dos comedores escolares. Tanto en España como en Portugal las cantidades registradas fueron superiores. Martins et al. (2014) obtuvieron 49,5 gramos en comedores escolares de escuelas primarias portuguesas. En España, Byker et al. (2014) obtuvo 164 gramos (34\% de la ración servida) de desperdicio en plato en menús de línea fría y 106 gramos ( $22 \%$ de la ración servida) en caliente; por otro lado, Derqui y Agustín (2016) ob- tuvieron en las cuatro escuelas primaria analizados en su estudio piloto, un desperdicio en plato promedio que va de 35 a 47 gramos; finalmente Derqui y Fernández (2017) probaron una herramienta estandarizada de auto-evaluación del desperdicio en cuatro escuelas de Barcelona y obtuvieron entre 40 y 100 gramos de desperdicio en plato promedio.

En respuesta a la alta variabilidad encontrada en estudios de desperdicio alimentario, se vienen desarrollando investigaciones que estudian las condicionantes del desperdicio desde diversos enfoques y bajo distintos niveles de análisis. Los estudios de desperdicio en comedores escolares suelen abordar las condicionantes a nivel de centro educativo, mientras que los que abarcan toda la fase de consumo de alimentos realizan un análisis a nivel del comportamiento de los individuos frente al desperdicio. El Programa de Acción de Residuos y Recursos Cordingley, Reeve y Stephenson (2011) considera que el desperdicio en plato en comedores escolares puede deberse a razones operativas, circunstanciales o de comportamiento. Derqui y Agustín 
(2016) además de las razones antes mencionadas, incluyen el enfoque institucional respecto al desperdicio. En la Tabla 2 se detallan las razones ya mencionadas, incluyendo factores que influyen en el desperdicio en plato por cada una de ellas.

Aranceta Bartrina et al. (2004); Priefer, Jörissen y Bräutigam (2016); Thyberg y Tonjes (2016) coinciden en que, de los factores mencionados en la Tabla 2, los que más inciden sobre el desperdicio alimentario en comedores escolares son el tamaño de las raciones, las características del menú-incluyendo los tipos de alimentos y la forma de presentacióny el grado de aceptación de los menús.

En relación a los factores de comportamiento, Bartrina et al. (2004) indican que los hábitos alimenticios en los comedores escolares están marcados por una clara preferencia por alimentos como pasta, arroz y patatas, y rechazo por el grupo de las verduras, legumbres y pescado. Rodriguez-Tadeo (2014) ven reflejadas estas preferencias en su investigación en comedores escolares en Murcia, al encontrar que en los primeros platos de línea caliente, las legumbres, ensaladas y guisos de pescado registraron las más altas proporciones de desperdicio, y en los segundos platos los hechos a base de aves, legumbres y pescado. Además de considerar la influencia del grado de aceptación de los alimentos sobre el desperdicio, Rodriguez-Tadeo (2014) encontraron también que los menús servidos en línea fría generan significativamente más desperdicios que los de línea caliente.

Continuando con factores asociados al comportamiento, Quested et al. (2013); Secondi, Principato y Laureti (2015) al analizar desperdicios a nivel del consumidor, señalan que los comportamientos y prácticas individuales asociadas con la generación de desperdicios alimentarios, pueden explicarse a partir de una relación compleja entre factores contextuales y factores propios de cada persona como actitudes, valores, motivaciones y hábitos.

Tabla 2. Causas y factores que influyen en el desperdicio en plato en comedores escolares (Derqui y Agustín, 2016; Cordingley, Reeve y Stephenson, 2011).

\begin{tabular}{cccc}
\hline \multicolumn{3}{c}{ Razones de la generación de desperdicios en plato en comedores escolares } \\
\hline Enfoque institucional & Operativas & Circunstanciales & De comportamiento \\
\hline Visión respecto a la & Menús: & Tiempo disponible & Preferencias o grados \\
orientación del comedor & Flexibilidad en el & para comer. & de aceptación por \\
escolar: Parte de una & tamaño de las raciones. & Condiciones de las & tipos de menú. \\
educación integral, o & Posibilidad de elección & instalaciones & Motivaciones externas \\
únicamente un servicio. & del menú. & Presencia de & Conciencia sobre el \\
& Formas de preparación & monitores & desperdicio \\
& y presentación de los & Tiempo entre el receso & \\
& alimentos. & y la hora de comer. & \\
\hline
\end{tabular}

Hebrok y Boks (2017); Thyberg y Tonjes (2016) coinciden en que se deben considerar los factores culturales, demográficos, socioeconómicos, políticos y geográficos que influyen sobre el comportamiento, en cada contexto. En los factores culturales se incluyen hábitos y actitudes frente al desperdicio, enfatizando en las diferencias entre países y dentro de cada país. En línea con lo anterior, Secondi, Principato y Laureti (2015) demuestran la existencia de una heterogeneidad del comportamiento frente al desperdicio al interior de los países de la Unión Europea, que no es explicada ni por las variables personales ni por las variables contextuales contempladas en su estudio, y que aún sigue sien- do poco explorada. Secondi, Principato y Laureti (2015), en un análisis de comportamiento frente al desperdicio desarrollado a nivel en la Unión Europea, confirmaron que las personas jóvenes tienden a generar más desperdicio que los mayores, y que las mujeres tienden a generar menos desperdicio que los hombres; en relación al grado de educación, encontró que personas que suspendieron su educación a una edad temprana tienden a desperdiciar menos alimentos; finalmente, respecto al lugar de residencia encontraron que personas que viven en ciudades medianas o grandes, desperdician más que los que viven en áreas rurales. 
En relación con los factores intrínsecos a cada persona, Hebrok y Boks (2017) indican que la mayoría de los consumidores no son conscientes de la comida que están desperdiciando, razón por la cual es necesario crear conciencia del problema para cambiar el comportamiento de los individuos (Quested et al., 2013). La información y educación son esenciales para influir en el comportamiento del consumidor, por esta razón Priefer, Jörissen y Bräutigam (2016) indican que las actividades de sensibilización deberían ser adaptadas a los diferentes grupos y deberían empezar en la infancia para tener un mayor efecto sobre el desperdicio.

Para evaluar la relación entre las actitudes de las personas y el desperdicio, Secondi, Principato y Laureti (2015) analizaron la presencia de hábitos y actitudes pro-ambientales como el reciclaje, y encontró que las personas con mayor nivel de compromiso ambiental y mayor consciencia sobre los impactos del desperdicio alimentario, tienden a tirar menores cantidades de comida.

\section{Metodología}

La investigación se enmarca desde una relación Universidad-Sociedad civil, que se articula en forma de la Cátedra Bancos de Alimentos UPMFESBAL, como un medio para establecer una colaboración estratégica a largo plazo, con el fin de "Trabajar con la gente", para conseguir un consumo racional y solidario. El marco metodológico se enmarca desde el modelo WWP (Cazorla, De Los Ríos y Salvo, 2013) de forma que la Cátedra Bancos de Alimentos se configura como una estructura estable y dinámica, que favorece la interacción de la Universidad con la sociedad, fomentando relaciones entre los distintos tipos de agentes y promoviendo el desarrollo de competencias desde la responsabilidad social y la transmisión de valores éticos. (De los Ríos et al., 2015). Desde este modelo resaltan las relaciones con distintos Colegios de España para acometer campañas de sensibilización y formación (De Los Ríos, Rodriguez y Pé, 2015).

La presente investigación analiza los desperdicios en plato en una muestra de comedores escolares en España. El desperdicio se mide en una muestra seleccionada, a partir de una metodología participativa diseñada por la Fundación PROCLADE, la Asociación PROYDE y la ONG SED. De acuerdo con los objetivos planteados en la investigación, se definen variables contextuales para identificar posibles condicionantes del desperdicio en plato. Finalmente, se realiza el procesamiento y análisis estadístico de los datos.

\subsection{Recolección de datos}

El trabajo de campo para la recolección de datos estuvo a cargo de la Fundación PROCLADE, la Asociación PROYDE y la ONG SED. La elección de los centros participantes respondió principalmente a la disposición de estos para realizar un inventario de desperdicios y participar activamente en el proceso de sensibilización e información a la comunidad educativa sobre los impactos sociales y ambientales de los desperdicios alimentarios. La muestra seleccionada fue de 118 centros educativos, ubicados en 14 comunidades autónomas de España.

La metodología de medición de desperdicios en comedores escolares tuvo un claro enfoque participativo. Los estudiantes se organizaron en grupos para realizar el conteo de comensales, la separación de desperdicios entre orgánico e inorgánico, y el pesado y registro de los grupos de desperdicios generados diariamente en cada comedor. Todas las actividades, si bien tuvieron como principales implicados a los estudiantes, fueron realizadas bajo la supervisión de educadores $u$ otras personas adultas encargadas del comedor.

Las mediciones de desperdicio en plato se realizaron diariamente de manera agregada no selectiva, en dos semanas no consecutivas. En el intervalo entre la primera y segunda medición, se realizaron actividades de sensibilización sobre el desperdicio alimentario con los docentes, personal del comedor escolar y niños y niñas. En la primera semana se realizaron 573 mediciones en 118 comedores escolares, mientras que en la segunda semana se realizaron 373 mediciones en 73 comedores escolares.

\section{Definición de variables del estu- dio}

Para la realización del análisis se empleó como variable dependiente el desperdicio promedio diario por comensal, obtenido a partir de las mediciones del desperdicio total por comedores escolares para cada día, descritas en el subapartado anterior, divididas entre el número de comensales. Como variables independientes se consideraron factores con- 
textuales a dos niveles-a nivel del territorio y del centro educativo-partiendo de lo encontrado en la literatura, así como los días de la semana.

A nivel del territorio, la selección de variables respondió a la necesidad de identificar nuevas condicionantes que contribuyan a explicar la heterogeneidad del comportamiento frente al desperdicio dentro de un país (Secondi, Principato y Laureti, 2015). Se consideraron como variables la ubicación de la provincia, la región y el tamaño de población donde está ubicado el centro educativo. Cabe resaltar que las dos primeras fueron empleadas en un estudio de equilibrio alimentario en comedores escolares de la Comunidad Valenciana, y presentaron diferencias respecto a la variedad de alimentos servidos (Llorens-Ivorra et al., 2017).

En la variable ubicación se diferencian los colegios ubicados en provincias de interior de los ubicados en provincias costeras. En la variable región se clasifican las comunidades autónomas en tres grandes regiones: Centro, Norte y MediterráneoCanarias. Finalmente, en la variable tamaño de población se clasifican los colegios de acuerdo con el tamaño de la población en la que se ubican, según la clasificación del Instituto Nacional de Estadística. En la Tabla 3 se clasifican los colegios y comensales en función de las variables contextuales mencionadas.

Tabla 3. Clasificación de los colegios y comensales en función de variables contextuales a nivel del territorio.

\begin{tabular}{|c|c|c|c|c|}
\hline Ubicación & Región & Población & Colegios & Comensales \\
\hline \multirow{13}{*}{ Interior } & \multirow{6}{*}{ Centro } & $<10.000$ & 42 & 1.156 \\
\hline & & $20.001-50.000$ & 1 & 16 \\
\hline & & $50.001-100.000$ & 15 & 566 \\
\hline & & $100.001-500.000$ & 3 & 364 \\
\hline & & $>500.001$ & 3 & 1.136 \\
\hline & & & 64 & 3.238 \\
\hline & Mediterráneo y Canarias & $100.0001-500.000$ & 2 & 164 \\
\hline & \multirow{6}{*}{ Norte } & $<1.000$ & 1 & 60 \\
\hline & & $10.001-20.000$ & 9 & 324 \\
\hline & & $20.001-50.000$ & 3 & 95 \\
\hline & & $100.0001-500.000$ & 21 & 1.132 \\
\hline & & & 34 & 1.611 \\
\hline & & & 100 & 5.013 \\
\hline \multirow{9}{*}{ Costa } & \multirow{4}{*}{ Mediterráneo y Canarias } & $20.001-50.000$ & 1 & 88 \\
\hline & & $100.0001-500.000$ & 2 & 317 \\
\hline & & $>500.001$ & 3 & 366 \\
\hline & & & 6 & 771 \\
\hline & \multirow{5}{*}{ Norte } & $10.001-20.000$ & 2 & 53 \\
\hline & & $20.001-50.000$ & 2 & 283 \\
\hline & & $100.0001-500.000$ & 3 & 278 \\
\hline & & & 7 & 614 \\
\hline & & & 13 & 1.385 \\
\hline
\end{tabular}

A nivel de centro educativo, se empleó el momento de medición-antes y después de una campaña de sensibilización-y el tipo de menú como variables independientes. La elección de la primera variable responde a la relación entre las actividades de sensibilización, la generación de conciencia sobre el desperdicio y la reducción del mismo (Priefer, Jörissen y Bräutigam, 2016; Quested et al., 2013). Para analizar la variable tipo de menú, se clasificaron los menús considerando la combinación de los compo- 
nentes principales del primer plato y segundo plato (Ver Tabla 4). Con el fin de facilitar la interpretación de resultados, se consideraron únicamente los menús que contaban con tres o más mediciones. Esta variable se incluye partiendo de la relación mencionada por Rodriguez-Tadeo (2014) entre el desperdicio en plato y el grado de aceptación o preferencias por tipos de alimentos en los comedores escolares.

Tabla 4. Componentes principales del primer y segundo plato considerados para definir tipos de menú.

\begin{tabular}{cc}
\hline \multicolumn{2}{c}{ Componentes principales del menú } \\
\hline Primer plato & Segundo plato \\
Crema/ puré de verduras & Pescado \\
Arroz/ pasta & Carnes \\
Sopas & Fritos \\
Legumbres & Huevos/ Tortilla \\
\hline
\end{tabular}

Finalmente, se consideraron también los días de la semana como una variable a analizar, con el objetivo de evaluar su incidencia sobre el desperdicio en plato generado.

\subsection{Análisis de datos}

El análisis estadístico de los datos se elaboró mediante el paquete estadístico Statgraphics XVII. Se realizó un análisis descriptivo del desperdicio en plato, y contrastes de hipótesis considerando $\mathrm{p}<0.05$ como significación estadística.

En primer lugar, se realizó un análisis descriptivo a las mediciones de desperdicio promedio diario por comensal de la primera semana en los 118 co- medores escolares seleccionados, con el objetivo de caracterizar los desperdicios en los comedores escolares. Conociendo la media y la desviación típica de los datos se identificaron los valores fuera de rangoaquellos separados de la media en más de dos desviaciones típicas-resultando en la exclusión de cinco comedores escolares de los análisis posteriores.

A continuación, se realizaron contrastes de hipótesis para cada una de las variables dependientes. Para definir las pruebas estadísticas a emplear -paramétricas o no paramétricas-, se evaluaron los valores de asimetría y curtosis. En la Tabla 5 se muestran las pruebas utilizadas para cada variable, considerando pruebas paramétricas para variables con valores de asimetría y curtosis entre -2 y 2 .

Tabla 5. Pruebas estadísticas por variable independiente.

\begin{tabular}{ccc}
\hline Variable independiente & Prueba paramétrica & Prueba no paramétrica \\
\hline Categórica con 2 niveles & tde Student & \\
- Momento de la medición & & \\
- Ubicación & & \\
Categórica con más de 2 niveles & & $H$ de Kruskal - Wallis \\
- Región & $H$ de Kruskal - Wallis \\
- Tamaño de población & $H$ de Kruskal - Wallis \\
- Días de la semana &
\end{tabular}

Para determinar las diferencias del desperdicio para las variables contextuales a nivel del territorio y el tipo de menú, se analizaron las 548 mediciones realizadas en 113 comedores escolares para la primera semana. Por otro lado, para determinar las diferencias del desperdicio entre antes y después de las actividades de sensibilización se excluyeron los comedores escolares que no contaban con ambas mediciones; además, con el fin de minimizar el efecto de la ubicación y región donde se encuentra 
el colegio se analizaron únicamente las 310 mediciones de los 63 comedores escolares de la región participantes y mediciones consideradas para cada centro. En la Figura 1 se detalla el flujo de colegios análisis.

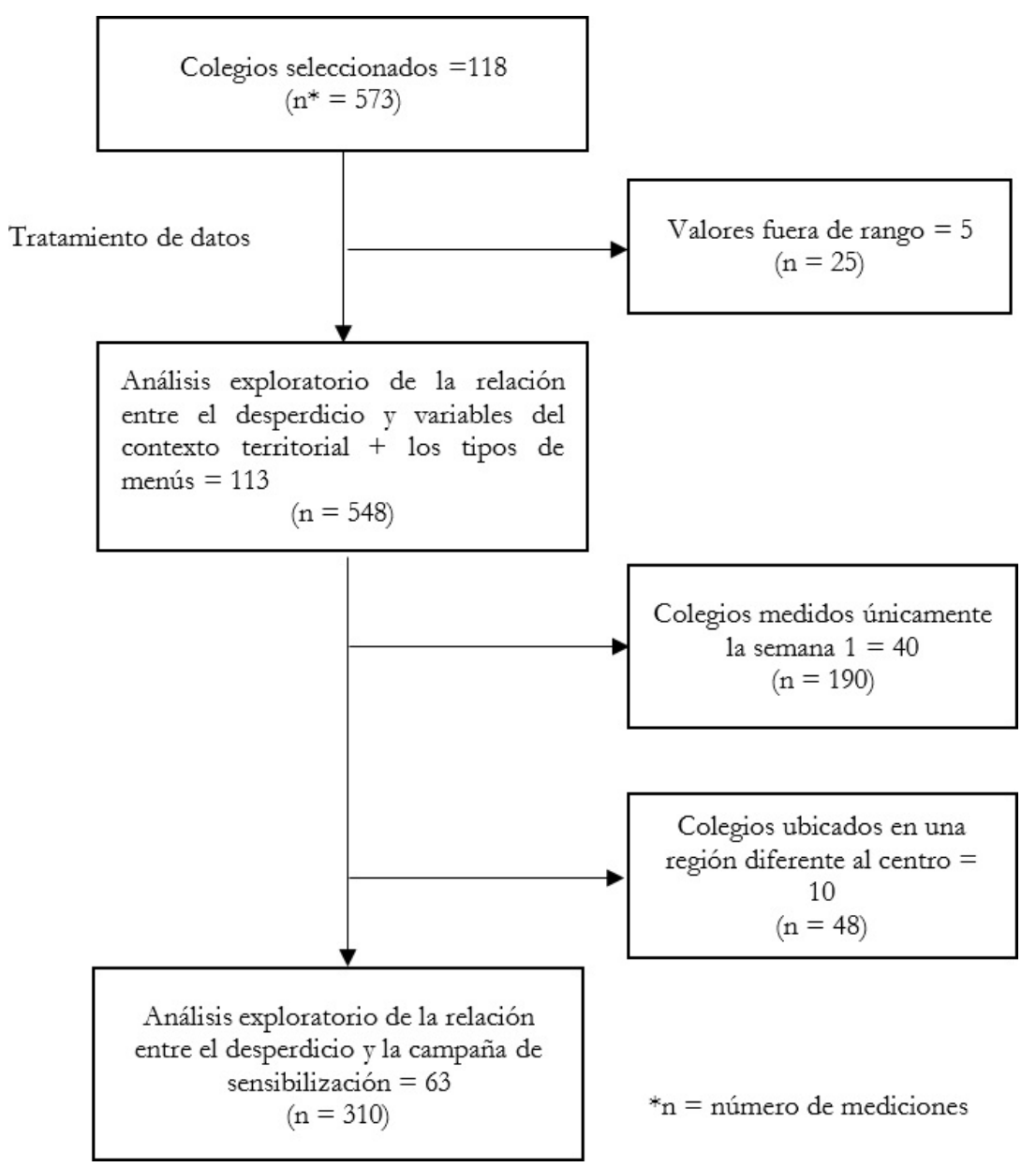

Figura 1. Flujo de colegios participantes y mediciones realizadas

Finalmente, se analizó la relación entre el desperdicio en plato y la suma de variables independientes. A nivel contextual se analizó la relación del desperdicio con ubicación-región; a nivel del centro educativo se analizó la relación con tipo de menúcampaña de sensibilización; también se analizó la relación de un factor territorial con un factor del centro educativo a partir de las variables tipo de menú-región; finalmente, se analizó el comportamiento semanal del desperdicio para las variables región, ubicación y campaña de sensibilización.

En todos los análisis mencionados no se probó la significación estadística de las diferencias del des- perdicio debido a la condición no paramétrica de los datos.

\section{Resultados}

En este apartado se presentan los resultados de la investigación. En un primer apartado se inicia la cuantificación del desperdicio diario promedio en plato y su comportamiento semanal; posteriormente se presenta la relación entre el desperdicio y las variables contextuales a nivel del territorio. Finalmente, se presenta la relación entre el desperdicio y las variables contextuales a nivel del centro educativo. 


\subsection{Cuantificación del desperdicio}

De las mediciones realizadas durante una semana en 113 comedores escolares, se obtuvo un desperdicio diario promedio por comensal de 76,61 gramos $( \pm 45,83)$. Al analizar el desperdicio por los días de la semana, se obtuvieron diferencias significativas (p-valor: 0,0023) entre los valores más altos de desperdicios - registrados el miércoles con 86,91 gra- mos $( \pm 61,27)$ y el viernes con 86,77 gramos $( \pm 61,93)$ - y el valor más bajo, registrado el jueves con 63,51 gramos $( \pm 48,33)$. En la Figura 2 se muestra una distribución asimétrica de los datos para todos los días de la semana, con una mayor dispersión de las mediciones hacia los valores más altos y valores atípicos en todos los días de la semana. Cabe resaltar que el coeficiente de variación del desperdicio por cada día va de 67 a 77 porciento.

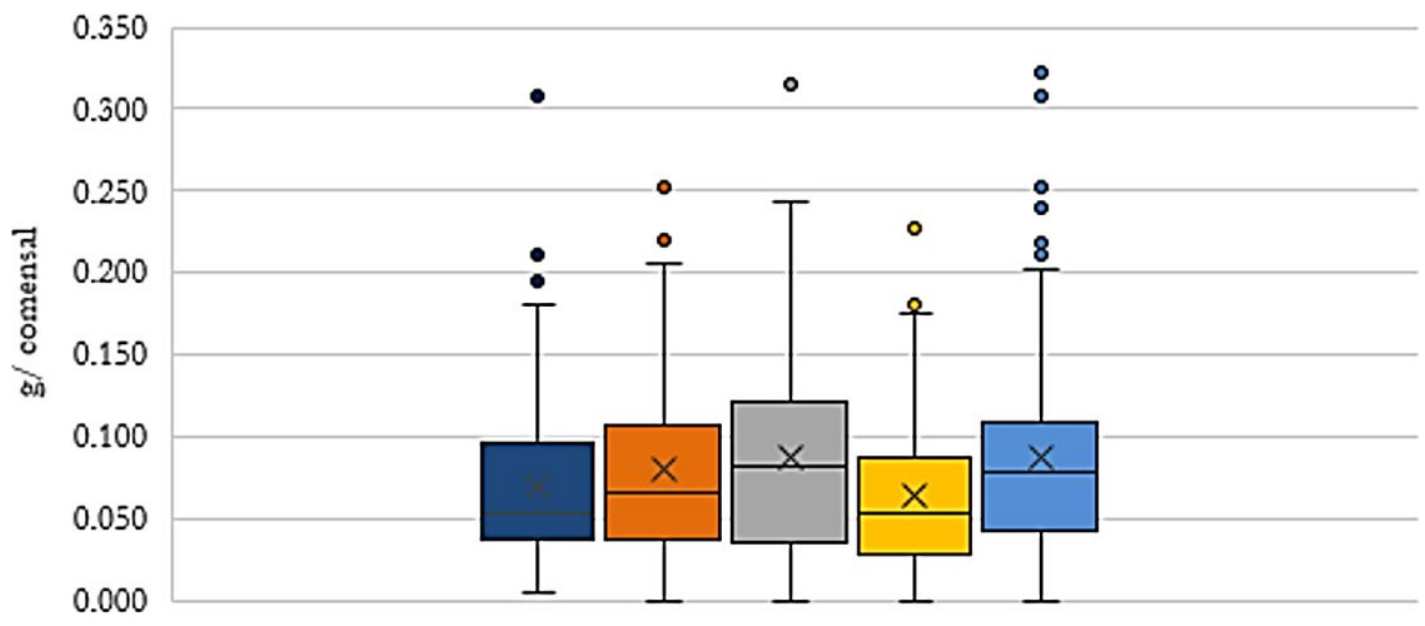

Días

Lunes $\square$ Martes $\square$ Miércoles $\square$ Jueves $\square$ Viemes

Figura 2. Comportamiento Semanal del Desperdicio Promedio diario (gramos/ comensal)

Nota: La caja incluye los datos ubicados entre el primer y tercer cuartil, la línea dentro de la caja representa la mediana y la (X) el promedio. Los bigotes presentan una longitud máxima de 1,5 veces la longitud de la caja; de presentarse valores fuera de esta distancia se consideran como valores atípicos, y se muestran como círculos.

\subsection{Relación entre el desperdicio en plato y variables contextuales a nivel del terri- torio}

Los contrastes de hipótesis indican que, de las variables contextuales consideradas a nivel del territorio, únicamente se encontraron diferencias significativas entre los desperdicios en plato de centros educativos ubicados en provincias de costa y de interior ( $\mathrm{p}$-valor $=0,039)$; en costa, el desperdicio diario promedio por comensal fue 101,15 gramos $( \pm 41,23)$ y en el interior 73,42 gramos $( \pm 45,20)$. En la Figura 3 se observa que los valores de desperdicio en interior presentan una distribución asimétrica con una mayor dispersión de las mediciones hacia los valores más altos, además de presentar valores atípicos; en cambio en la costa se observa una distribución más simétrica, aunque también se registra un valor atípico.

Por otro lado, tanto para la variable región como para tamaño de población, no se encontraron diferencias significativas en el desperdicio. Es importante resaltar que, en el análisis de las tres variables contextuales a nivel del territorio, el coeficiente de variación para la mayoría de los niveles de cada variable presenta valores altos, entre 40 y 70 por ciento. 


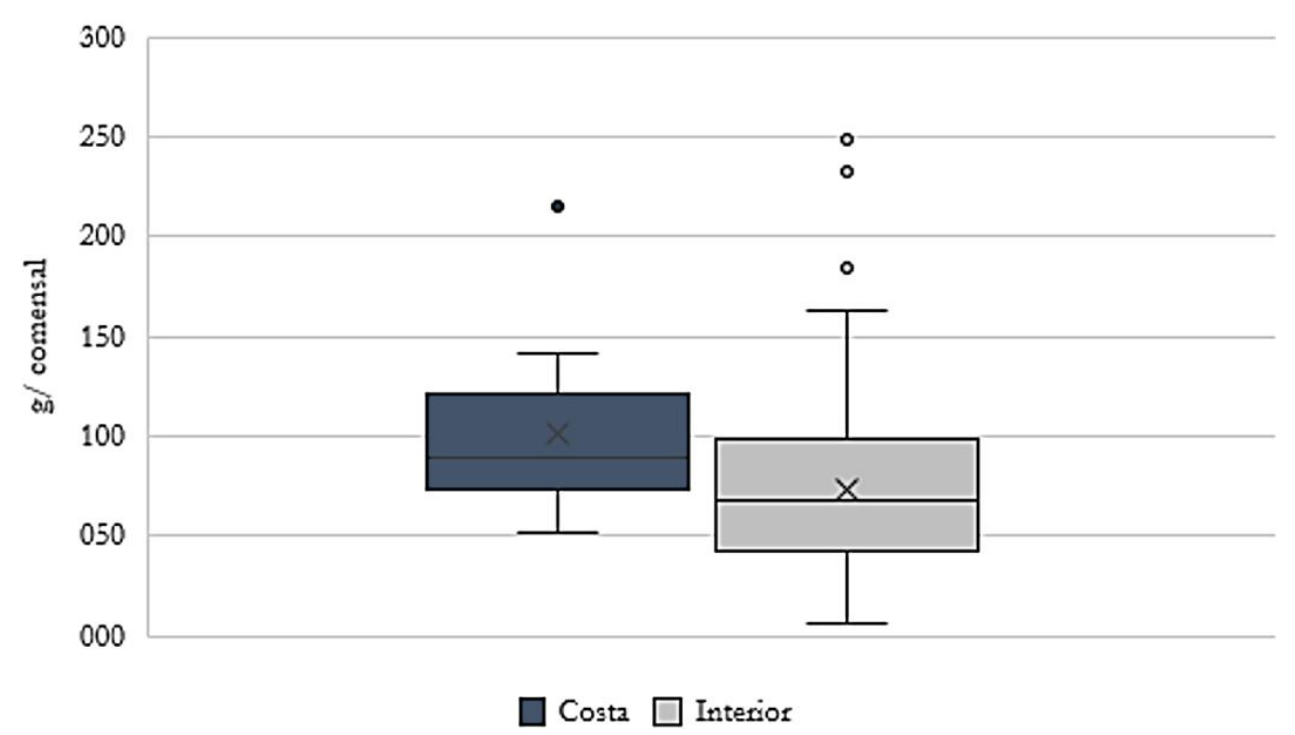

Figura 3. Desperdicio diario promedio por comensal (g) según ubicación Nota: Ver nota de la Figura 2.

Con el fin de comprobar si las diferencias significativas de desperdicio, identificadas entre comedores escolares de costa e interior, se observan tanto para la región del Mediterráneo-Canarias como para el Norte, se realizó un análisis de la relación entre la suma de las variables ubicación y región, y el desperdicio en plato. En la Figura 4 se observa que estas diferencias se cumplen para ambas regio- nes; la región del Mediterráneo-Canarias presenta un desperdicio promedio de 45,68 gramos $( \pm 21,68)$ en las provincias del interior contra 96,30 gramos $( \pm 28,86)$ en la costa, mientras que la región del Norte presenta un desperdicio promedio de 74,99 gramos $( \pm 46,01)$ en el interior contra 105,31 gramos $( \pm 49,05)$ en la costa.

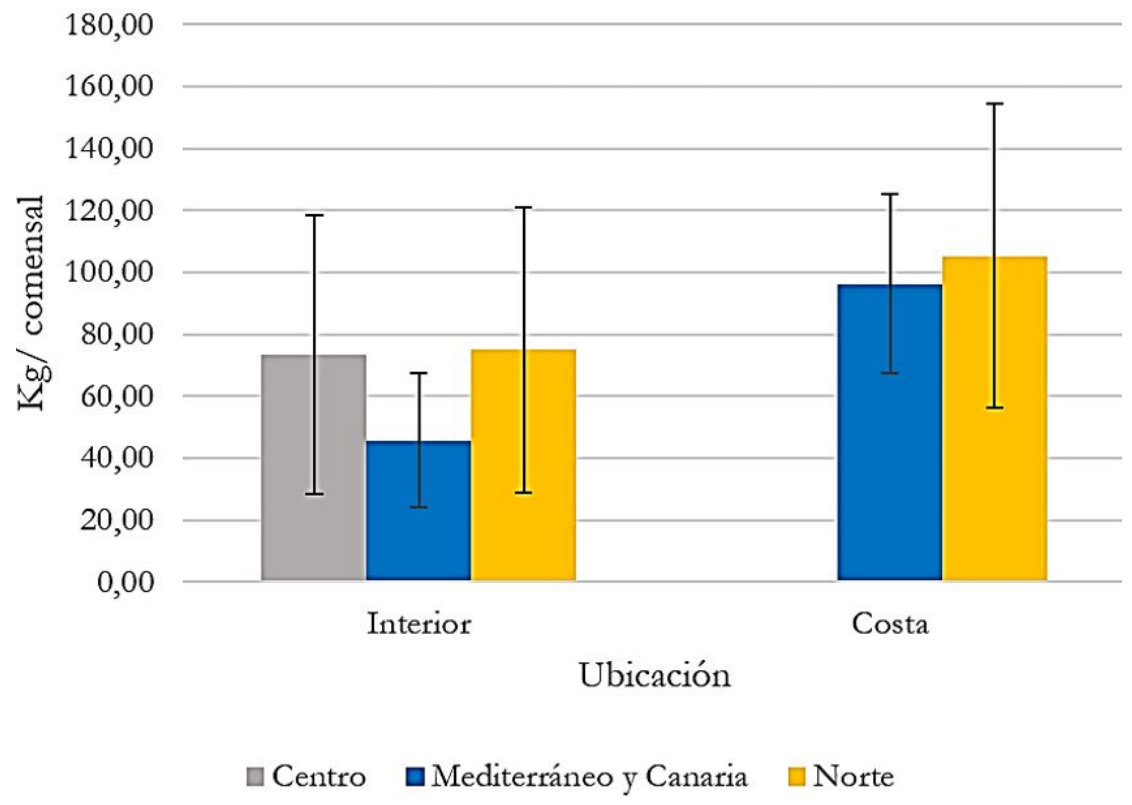

Figura 4. Desperdicio diario promedio por comensal según ubicación y región del centro educativo. 
En el análisis del comportamiento semanal del desperdicio diario promedio según la ubicación de los centros educativos, se encontró que los comedores escolares ubicados en la costa tienden a generar cantidades similares de desperdicio todos los días de la semana, mientras que en el interior la tendencia indica que el miércoles y el viernes se generan más desperdicios y el jueves registra los valores más bajos (ver Figura 5).

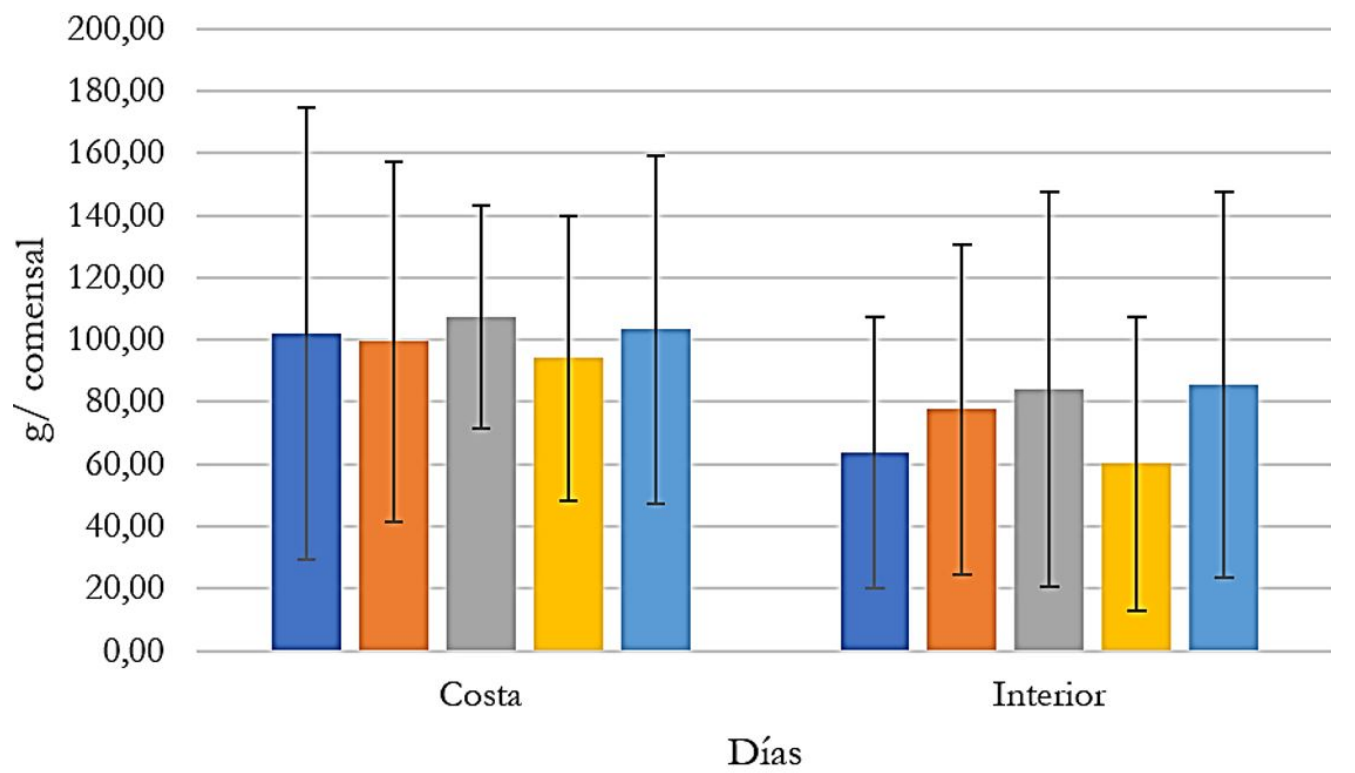

\section{$\square \mathrm{L} \square \mathrm{M} \square \mathrm{X} \square \mathrm{J} \square \mathrm{V}$}

Figura 5. Comportamiento semanal del desperdicio promedio diario por ubicación.

Con el fin de identificar si el comportamiento semanal del desperdicio en colegios de costa e interior es el mismo en las regiones que conforman ambos grupos, se realizaron dos análisis - uno para costa y otro para interior -, del comportamiento semanal del desperdicio por región (ver Figura 6). En la costa no se observan claras diferencias en el comportamiento semanal de los desperdicios entre las re- giones del Norte y del Mediterráneo-Canarias, presentando ambas un comportamiento uniforme del desperdicio en la semana. Por otro lado, en el interior las diferencias son más claras; el centro presenta un comportamiento uniforme del desperdicio en la semana, mientras que el norte registra los valores más altos de desperdicio el miércoles y viernes, y el valor más bajo el jueves. 

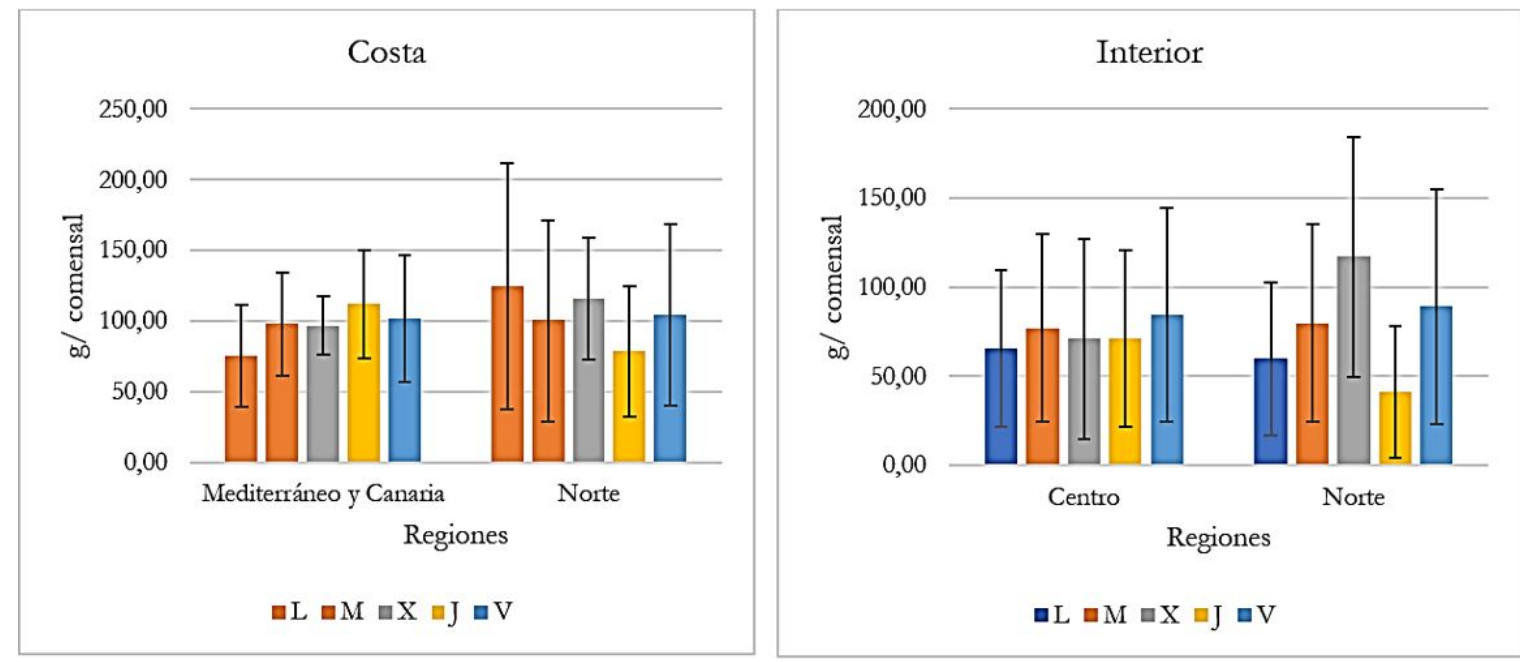

Figura 6. Comportamiento Semanal del Desperdicio Promedio Diario (g/ comensal) según Ubicación y Región.

\subsection{Relación entre el desperdicio en plato y variables contextuales a nivel del centro educativo}

A nivel del centro educativo, una de las variables analizadas fue el tipo de menú. En la Figura 7 se muestra la frecuencia de cada tipo de menú para las 548 mediciones realizadas en la primera semana, diferenciado por regiones. En la región centro, 3 de los 11 tipos de menús ofrecidos cubren la mitad de las mediciones analizadas, mientras que en la región
Mediterráneo-Canarias son 4 de 12 y en el norte 2 de 10. En el centro, los menús más frecuentes son legumbres/ carnes, seguido por arroz-pasta/ pescado y legumbres/ pescado. En el Mediterráneo y Canarias el menú de arroz-pasta/ pescado es también de los más frecuentes, seguido de arroz-pasta / carnes, puré o crema de verduras/ carnes y sopa/ carnes. Finalmente, la región norte a diferencia de las anteriores presenta menos variabilidad en los tipos de menús, siendo arroz-pasta / carnes el menú más frecuente con $37 \%$, seguido por sopa/ carnes. 


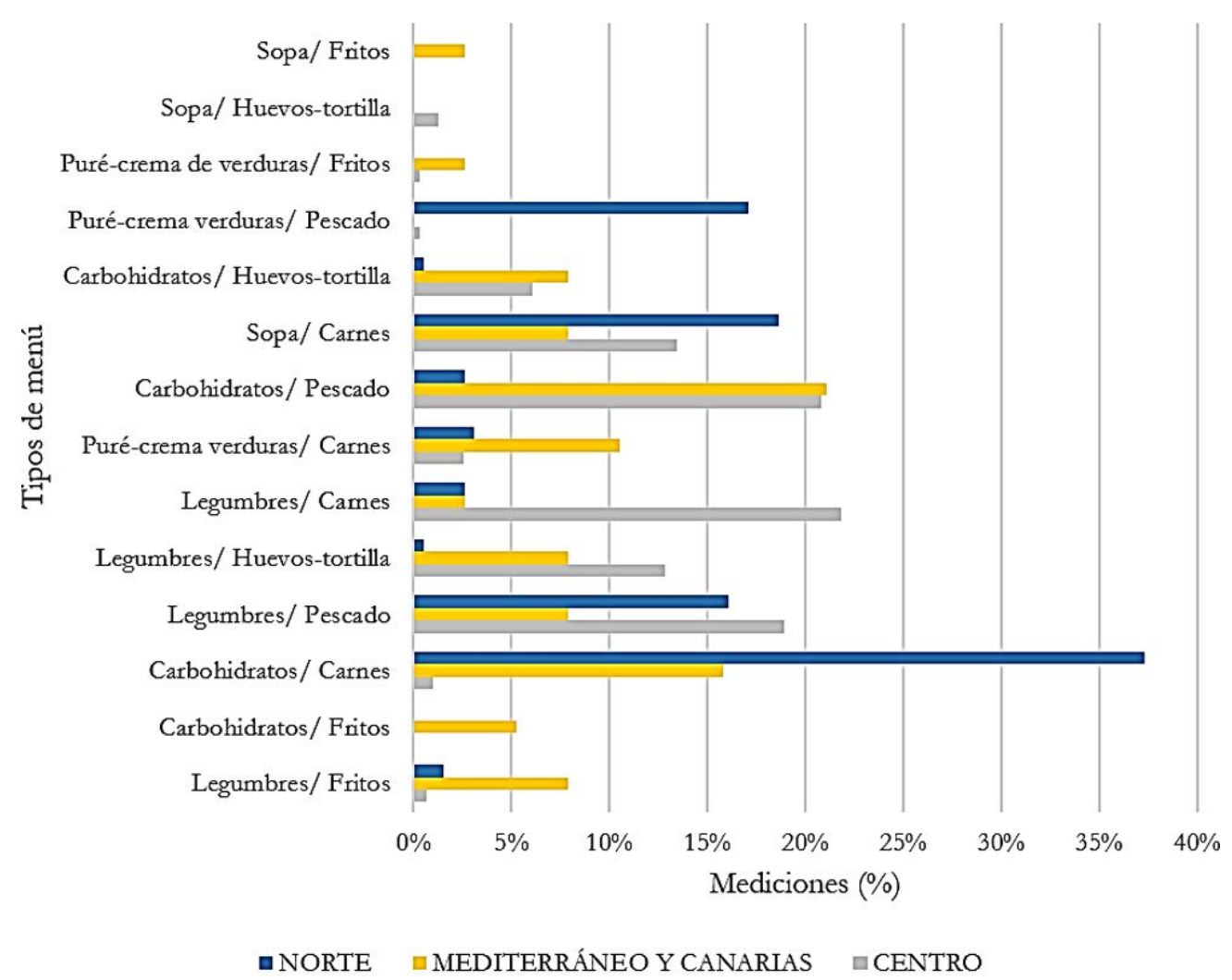

Figura 7. Frecuencia (\%) de cada tipo de menú por regiones.

Nota: La frecuencia de cada tipo de menú se refiere a cantidad relativa de veces que se registra un tipo de menú, considerando como total las 548 mediciones realizadas en 113 comedores escolares, durante una semana.

La relación entre el tipo de menú y el desperdicio diario promedio por comensal varía por regiones, de acuerdo con lo presentado en la Figura 8. Para la región centro, los menús que tienen carne como principal componente del segundo plato son los que más desperdicios generan, mientras que los que incluyen huevos-tortilla registran menos desperdicios; respecto al primer plato, los mayores desperdicios se generan con arroz-pasta, independientemente del segundo plato. Para la región norte en cambio, la tendencia del desperdicio se ve marcada por el componente principal del primer plato, siendo los platos con legumbres los que más desperdicios generan, seguidos de los platos con arroz-pasta, y finalmente platos con puré o crema de verduras y sopas. Finalmente, para la región del Mediterráneo y Canarias no se observa una tendencia clara, debido principalmente al bajo número de repeticiones por plato en todos los casos. 

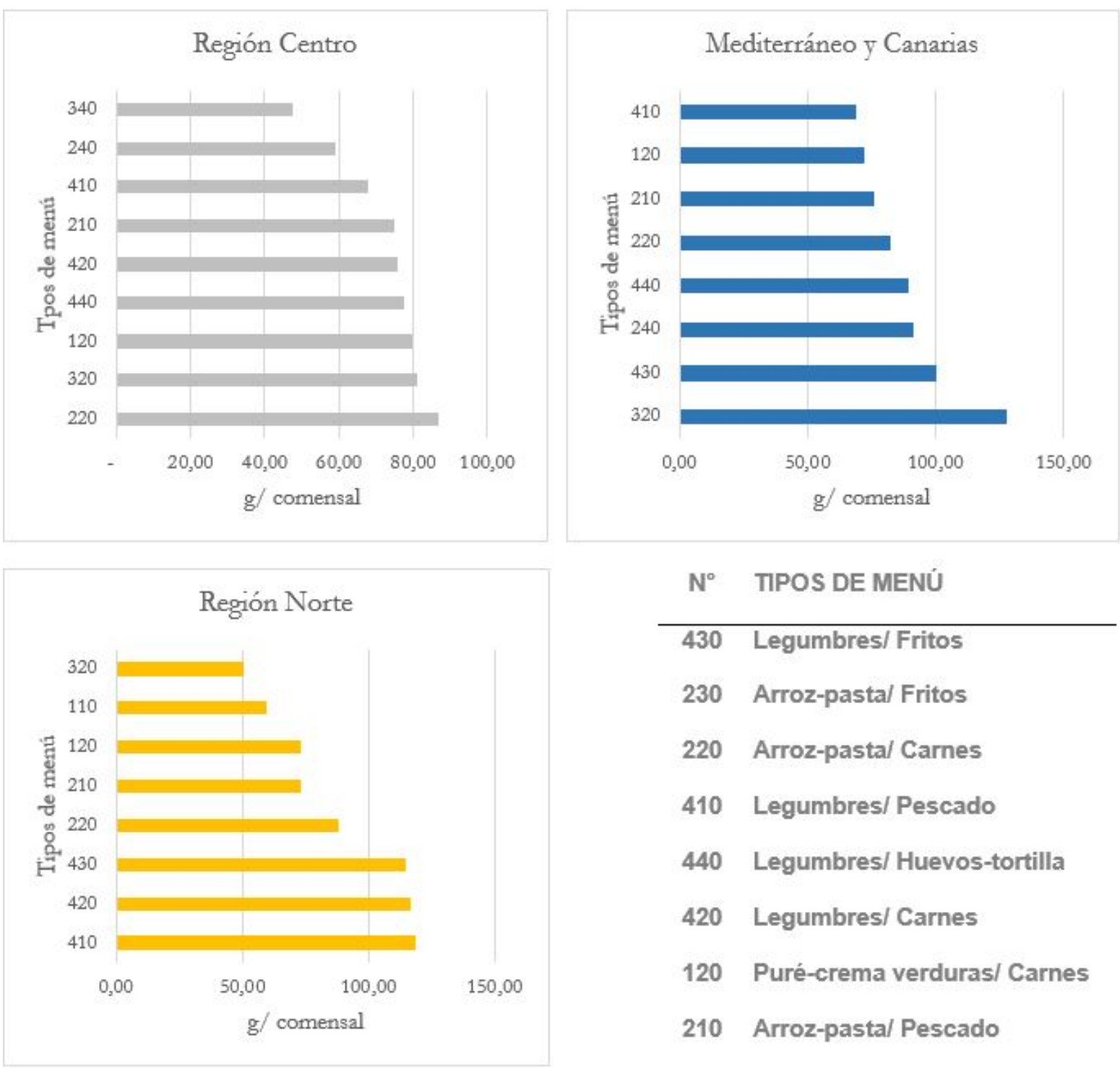

$\mathbf{N}^{\circ} \quad$ TIPOS DE MENÚ

\begin{tabular}{ll}
\hline 430 & Legumbres/ Fritos \\
230 & Arroz-pasta/ Fritos \\
220 & Arroz-pasta/ Carnes \\
410 & Legumbres/ Pescado \\
440 & Legumbres/ Huevos-tortilla \\
420 & Legumbres/ Carnes \\
120 & Puré-crema verduras/ Carnes \\
210 & Arroz-pasta/ Pescado \\
320 & Sopa/ Carnes \\
240 & Arroz-pasta/ Huevos-tortilla \\
\hline 110 & Puré-crema verduras/ Pescado \\
\hline 340 & Puré-crema de verduras/ \\
\hline 130 & Fritos \\
\hline 330 & Sopal Huevos-tortilla \\
\hline
\end{tabular}

Figura 8. Desperdicio promedio diario (g/ comensal) según tipo de menú. 
La otra variable analizada fue el momento de medición de los desperdicios, correspondiente con antes y después de una campaña de sensibilización. Para los 63 colegios analizados en la región centro, existen diferencias significativas ( $p$-Valor: 0.00007) entre el desperdicio promedio medido antes y después de una campaña de sensibilización (Ver Figura 9). Los datos de desperdicio en plato, tanto antes como después de la campaña de sensibilización, presentan distribución asimétrica con una mayor dispersión de las mediciones hacia los valores más altos. El promedio en la primera semana fue 72,67 gramos $( \pm 45,31)$ y en la segunda 57,64 gramos $( \pm 36,74)$, lo que indica una reducción de $20,7 \%$ en la generación de desperdicios.

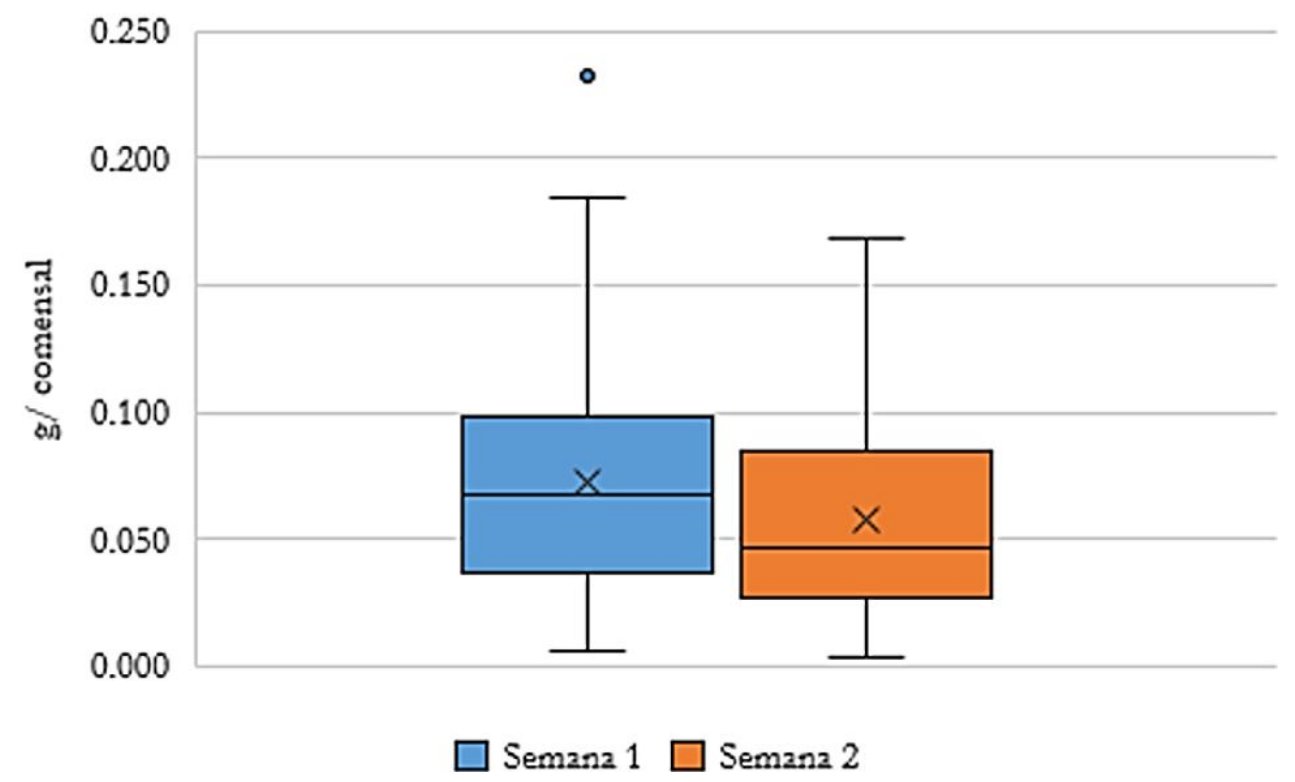

Figura 9. Desperdicio diario promedio (g/ comensal) antes y después de una campaña de sensibilización. Nota: Ver nota de la Figura 2.

El comportamiento semanal del desperdicio difiere según el momento de la medición. En la Figura 10 se observa que, si bien en ambas semanas se mantiene la tendencia de desperdiciar más el viernes, los desperdicios son más constantes a lo largo de la semana dos. El coeficiente de variabilidad de los desperdicios diarios respecto a la media semanal en la semana uno es $9,81 \%$, mientras en la semana dos es $7,52 \%$. 

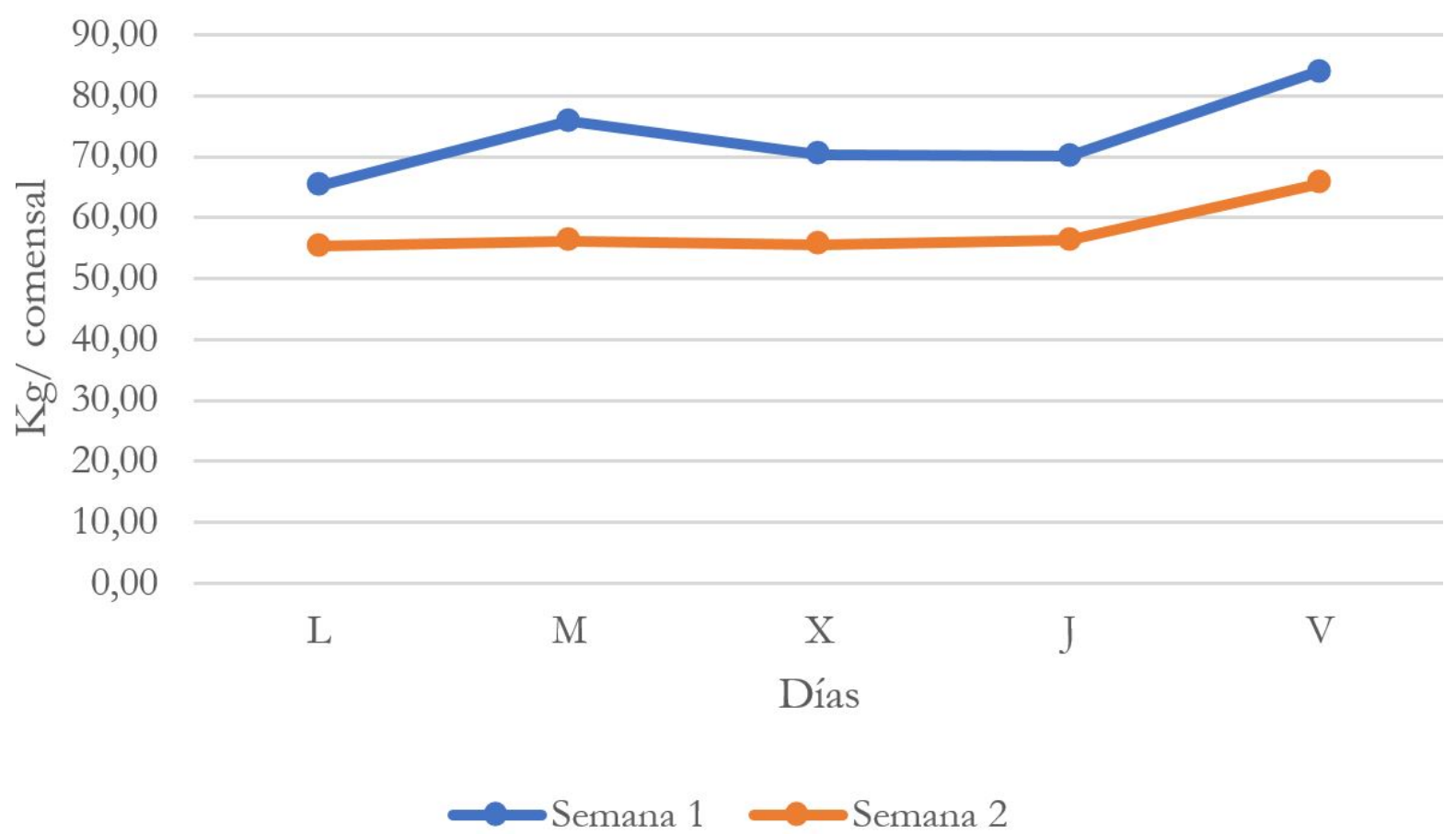

Figura 10. Comportamiento semanal del desperdicio promedio diario, antes y después de una campaña de sensibilización.

De forma general, los desperdicios generados por cada tipo de menú en la región centro tienden a disminuir de la semana uno a la semana dos, tal como se observa en la Figura 11. La mayor reducción de desperdicios se presenta en los menús: purécrema de verduras/carne con 56\%, sopa/huevos- tortilla con $49 \%$ y legumbres/huevos-tortilla con $38 \%$. Cabe resaltar que los dos tipos de menú que incluyen pescado como segundo plato son los únicos que presentan un incremento en el desperdicio de la primera a la segunda semana: legumbres/ pescado con $27 \%$ y arroz-pasta/ pescado con $2 \%$. 


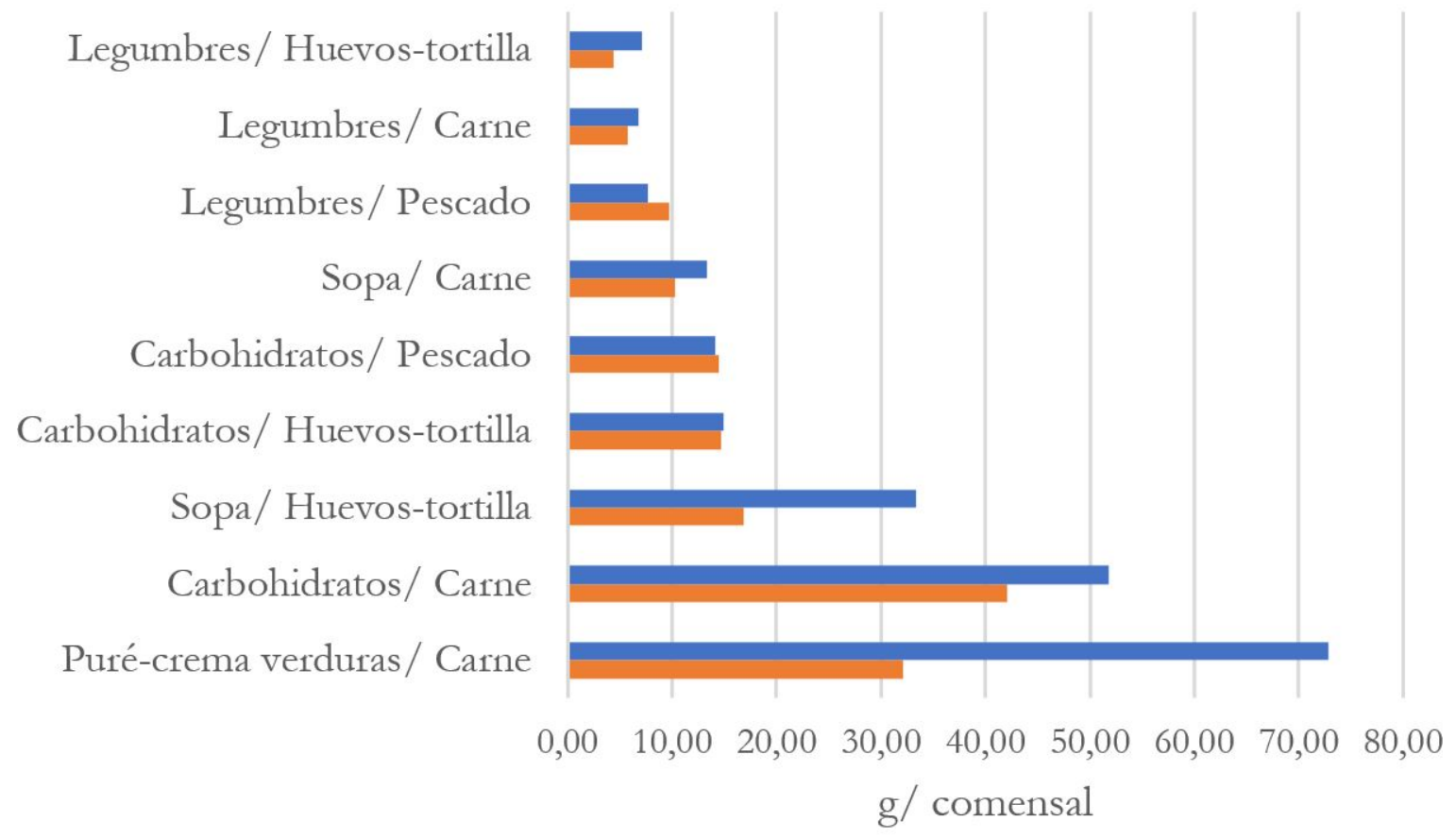

\section{Semana 1 Semana 2}

Figura 11. Desperdicio promedio diario (g/comensal) por tipo de menú, antes y después de las actividades de sensibilización.

Al tomar en consideración la frecuencia con la que son servidos los platos (ver Figura 12) antes y después de la campaña de sensibilización, resulta que dos de los tres menús más frecuentes-arrozpasta/ pescado y legumbres/ pescado-son también los que registran un incremento en el desperdicio entre la semana uno y dos. Por otro lado, los tres menús que registran según la Figura 11 mayor disminución de desperdicio, no son servidos frecuentemente.

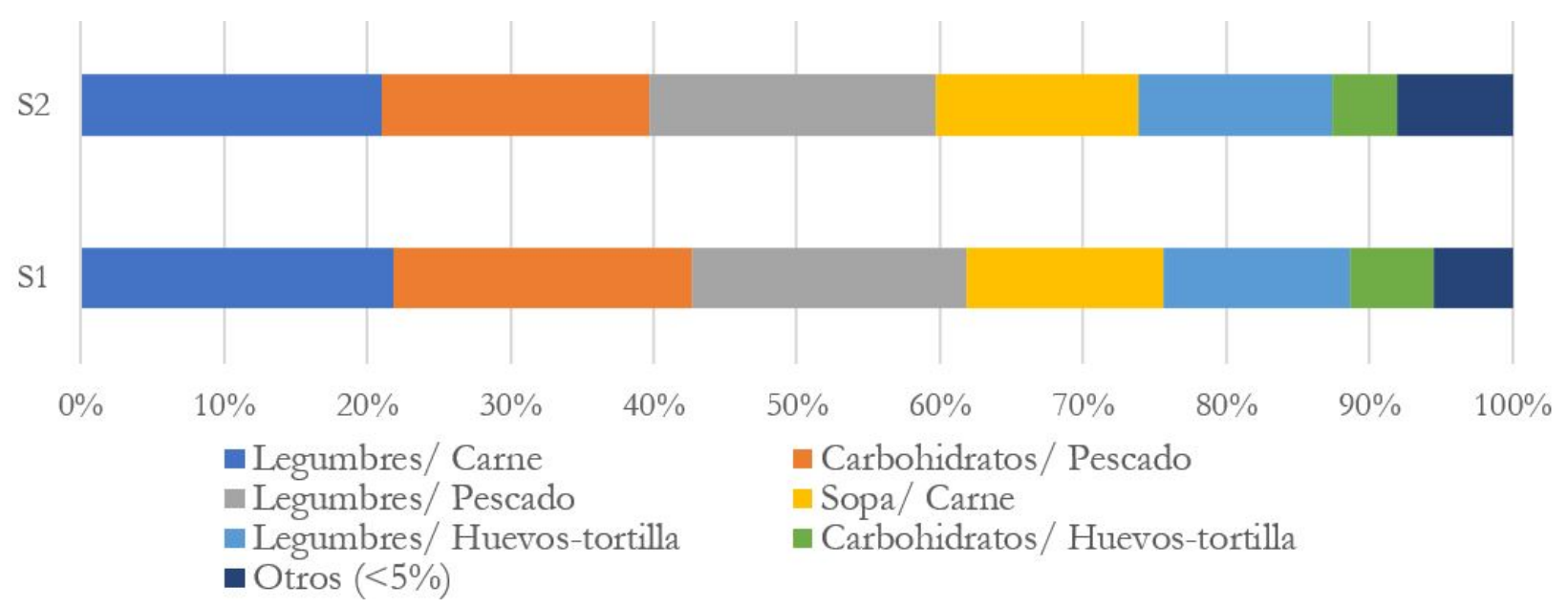

Figura 12. Frecuencia (\% días) de menús antes y después de las actividades de sensibilización. 


\section{Discusiones}

Para prevenir y/o reducir el desperdicio alimentario, el Ministerio de Agricultura y Pesca, Alimentación y Medio Ambiente (2017) en su estrategia Más alimentos, menos desperdicios indica que es necesario realizar estudios que respondan a cuatro interrogantes clave: cuánto se desperdicia, dónde ocurre el desperdicio, cómo ocurre y cuáles son las posibles causas o condicionantes del mismo. Considerando la importancia del desperdicio en el sector de restauración, la presente investigación se centra en el desperdicio en plato generado en comedores escolares en España. Los resultados obtenidos brindan una primera aproximación sobre la cantidad y los condicionantes de la heterogeneidad de este desperdicio, a partir de un análisis de la relación entre el desperdicio en plato y factores contextuales a nivel del territorio y de los centros educativos.

En los comedores escolares analizados se registró un desperdicio promedio diario por comensal de 76,62 gramos, valor que entra dentro del amplio rango encontrado en estudios realizados en comedores escolares en España (Derqui y Agustín, 2016; Derqui y Fernández, 2017; Rodriguez-Tadeo, 2014). Cabe resaltar que esta investigación, a diferencia de los estudios mencionados, ha trabajado con una muestra de colegios ubicados en distintas comunidades autónomas del territorio español que, si bien no es representativa del país, nos brinda una idea más aproximada a la cantidad de desperdicio generado en este sector.

El desperdicio de alimentos depende de una serie de factores que deben ser analizados considerando el contexto. Hebrok y Boks (2017) indican, en su estudio de comportamiento del consumidor frente al desperdicio en países de la Unión Europea, que aún se conoce poco sobre cuánto de la heterogeneidad del desperdicio es atribuible a factores contextuales. La presente investigación clasificó los factores contextuales en factores vinculados al centro educativo, y factores vinculados al territorio, y analizó la relación de ambos con el desperdicio en plato generado en comedores escolares.

A nivel del territorio, la única variable que mostró diferencias significativas en el desperdicio en plato fue la ubicación de los colegios, registrando valores más altos de desperdicio en provincias de costa que en provincias de interior. La presente investigación evidencia que existe una relación entre el desperdicio en plato y la ubicación de los cole- gios; sin embargo, para vincular el desperdicio en plato con factores de comportamiento asociados a la ubicación en costa o interior, sería necesario explorar variables de actitud, valores y hábitos frente al desperdicio, que ocasionan la heterogeneidad del desperdicio entre ubicaciones.

A nivel de centro educativo, Derqui y Fernández (2017) indican que la generación de desperdicios en plato depende de la receptividad de los comensales frente a los alimentos; según Cordingley, Reeve y Stephenson (2011) parte de esta receptividad depende de factores de comportamiento, dentro de los cuales se abordan el grado de aceptación de los menús y el nivel de conciencia de los niños frente al desperdicio. Para brindar un primer acercamiento a estos factores de comportamiento, se analizó el tipo de menú ofertado y la presencia de actividades de sensibilización en los centros educativos.

El grado de aceptación de los menús ha sido descrito por Bartrina et al. (2004). En su estudio sobre los hábitos alimenticios de comedores escolares en España, se indica la preferencia por arroz-pasta, y el rechazo por verduras, legumbres y pescado. En la presente investigación, la discriminación por regiones matiza las observaciones del estudio anteriormente citado, y da indicios de la influencia del contexto territorial sobre el grado de aceptación del menú. En la región norte se cumple que los primeros platos con legumbres son los que generan más desperdicios. Sin embargo, en la región centro se encontró que los menús con carne como segundo plato generan más desperdicios que los menús con pescado, y que el componente que genera más desperdicios en el primer plato son los arroz-pasta, contrariamente a lo descrito por Bartrina et al. (2004).

El análisis del desperdicio en plato que considera como variable territorial la región - Norte, Centro y Mediterráneo-Canarias - no presentó diferencias significativas. Sin embargo, tal como se detalla en el párrafo anterior, este factor contextual muestra una relación con el grado de aceptación de los menús, y este a su vez influye en el nivel de desperdicio en plato por cada tipo de menú.

Para obtener resultados más claros respecto a este factor, se recomienda realizar estudios que incluyan más repeticiones por tipo de menú, y que de preferencia permitan cuantificar el desperdicio por tipo de alimento, para de esta manera analizar las posibles razones de la mayor o menor aceptación de un menú. Finalmente, para tomar medidas que influyan sobre este factor, es importante evaluar otras 
características del menú, como por ejemplo la forma de preparación y presentación, la temperatura a la que es servido, entre otros.

La importancia de las actividades de sensibilización para la creación de conciencia frente al desperdicio es descrita por Priefer, Jörissen y Bräutigam (2016). En el presente estudio se encontraron diferencias significativas entre el desperdicio medido antes y después de las actividades de sensibilización para la región centro, lo que nos indica que existe una relación entre estas actividades y la reducción del desperdicio. Sin embargo, cabe resaltar que para establecer una relación entre la sensibilización y la creación de conciencia frente al desperdicio se deberían considerar otras variables de comportamiento, valores y/o hábitos de los individuos frente al desperdicio, a mediano y/o largo plazo.

$\mathrm{Al}$ analizar la relación entre las actividades de sensibilización y el desperdicio por tipo de menú, se encontró que únicamente los menús con legumbres y/o pescado no presentaron reducción en el desperdicio. El rechazo por este tipo de alimentos, descrito por Bartrina et al. (2004) podría explicar este fenómeno, ya que la creación de conciencia sobre el desperdicio no tiene porqué influir en las preferencias por cierto tipo de alimentos.

Finalmente, al analizar la relación entre los días de la semana y el desperdicio diario en plato, no se encuentra una tendencia clara. Al incluir las variables contextuales de ubicación y región en el análisis del comportamiento semanal del desperdicio, se encuentra que únicamente los comedores escolares de las provincias de interior en la región norte presentan diferencias significativas entre los días, lo que podría explicarse por el efecto de variables contextuales que varían en función de los días, como el tipo de menú.

También se encontró que el comportamiento semanal del desperdicio después de las actividades de sensibilización es más uniforme a lo largo de la semana. Esto puede deberse a la relación de la generación de conciencia sobre el desperdicio y otros factores que causan valores altos de desperdicio algún día de la semana, respecto al promedio semanal.

\section{Conclusiones}

La presente investigación demuestra que los factores contextuales a nivel del centro educativo y a nivel del territorio están estrechamente relacionados entre ellos, e influyen sobre el desperdicio en plato. A nivel del territorio se encontró que los desperdicios en plato entre colegios ubicados en provincias de costa y provincias de interior muestran diferencias significativas, registrándose en costa los valores más altos de desperdicio. A nivel del centro educativo se encontraron también diferencias significativas entre los desperdicios medidos antes y después de las campañas de sensibilización, presentándose una reducción en el desperdicio después de las campañas. Finalmente, si bien los desperdicios en plato en las tres regiones analizadas-Centro, Norte, Mediterráneo-Canarias-no muestran diferencias significativas entre ellos, al analizar la cantidad de desperdicio en plato registrado por cada tipo de menú, se encontró que estos valores difieren según la región en la que está ubicado el colegio, lo que da indicios de la relación entre la región y el grado de aceptación o preferencias de los estudiantes por cada tipo de menú. Para conocer el efecto de estos factores sobre el desperdicio en plato, se recomienda analizar variables de comportamiento que brinden mayor claridad sobre las relaciones entre factores condicionantes contextuales, comportamiento de individuos o de un colectivo frente al desperdicio y finalmente la cantidad de desperdicio en plato.

El principal objetivo de estudiar el comportamiento frente al desperdicio alimentario en comedores escolares es diseñar medidas de reducción y/o prevención. Para esto se recomienda, en primer lugar, continuar explorando variables relacionadas al contexto, que contribuyan a explicar la variabilidad de los datos de desperdicio promedio diario en comedores escolares; y finalmente, con los condicionantes identificados, realizar un modelo que vincule los condicionantes a nivel contextual e individual, y permita conocer en qué medida cada uno influye sobre el desperdicio.

\section{Referencias}

Bartrina, J. Aranceta, C. Pérez Rodrigo, L. Serra Majem y A. Delgado Rubio. 2004. "Hábitos alimentarios de los alumnos usuarios de comedores escolares en España. Estudio «Dime Cómo Comes»." Atención Primaria 33(3):131-139. Online: https:/ / doi.org/10.1016/S0212-6567(04)79373-7.

Buzby, Jean C, Joanne F Guthrie et al. 2002. "Plate waste in school nutrition programs." Electronic Publications from the Food Assistance \& Nutri- 
tion Research Program 36(2):220-238. Online: https: //goo.gl/oNxpVK.

Byker, Carmen J., Alisha R. Farris, Michael Marcenelle, George C. Davis y Elena L. Serrano. 2014. "Food Waste in a School Nutrition Program After Implementation of New Lunch Program Guidelines." Journal of Nutrition Education and Behavior 46(5):406-411. Online: https://doi.org/10.1016/ j.jneb.2014.03.009.

Cazorla, Adolfo, Ignacio De Los Ríos y Miguel Salvo. 2013. "Working With People (WWP) in rural development projects: A proposal from social learning." Cuadernos de desarrollo rural 10(spe70):131-157. Online: https://goo.gl/ Fqq5cG.

Cordingley, F, S Reeve y J. Stephenson. 2011. Food waste in schools. Technical report Waste and Resources Action Programme. Online: https://goo. $\mathrm{gl} / \mathrm{kacM} 5 \mathrm{G}$.

De los Ríos, I, A Cazorla, S Sastre y C. Cadeddu. 2015. New university-society relationships for rational consumption and solidarity: actions from the Food Banks-UPM Chair. In Envisioning a future without food waste and food poverty. Wageningen Academic Publisher. Online: https://goo.gl/ DTifaF.

De Los Ríos, Ignacio, Fernando Rodriguez y Cristina Pé. 2015. "Promoting Professional Project Management Skills in Engineering Higher Education: Project-Based Learning (PBL) Strategy*." International Journal of Engineering Education 31:115. Online: https://goo.gl/hZEJi5.

Derqui, B. y A Agustín. 2016. Estudio piloto para la Medicion y Reduccion del Desperdicio de Alimentos en Comedores Escolares: Auditoria y Autoevaluacion. Technical report Catálogo de Publicaciones de la Administración General del Estado. Online: https://goo.gl/bc6FNX.

Derqui, BelÃ@)n y Vicenc Fernández. 2017. “The opportunity of tracking food waste in school canteens: Guidelines for self-assessment." Waste Management 69:431-444. Online: https: / / doi.org/10. 1016/j.wasman.2017.07.030.

Engström, Rebecka y Annika Carlsson-Kanyama. 2004. "Food losses in food service institutions Examples from Sweden." Food Policy 29(3):203 -
213. Online: https://doi.org/10.1016/j.foodpol. 2004.03.004.

Eriksson, Mattias, Christine Persson Osowski, Christopher Malefors, Jesper Björkman y Emelie Eriksson. 2017. "Quantification of food waste in public catering services A case study from a Swedish municipality." Waste Management 61:415422. Online: https://goo.gl/Yn6aoS.

FAO. 2011. Global food losses and food waste Extent, causes and prevention. In Food and agriculture organization of the United Nations. Online: https://goo.gl/Ak6Gwr.

FAO. 2014. Mitigation of food wastage: Societal costs and benefits. Technical report Food and agriculture organization of the United Nations. Online: https:/ /goo.gl/CofuLw.

FESBAL. 2017. Memoria Anual 2017. Technical report FESBAL. Online: https://goo.gl/TL7b4p.

Hebrok, Marie y Casper Boks. 2017. “Household food waste: Drivers and potential intervention points for design - An extensive review." Journal of Cleaner Production 151:380 - 392. Online: https://doi.org/10.1016/j.jclepro.2017.03.069.

HLPE. 2014. Food Losses and Waste in the Context of Sustainable Food Systems. Technical report A report by the High Level Panel of Experts on Food Security and Nutrition of the Committee on World Food Security. Rome. Online: https://goo.gl/1S1eQF.

Lipinski, B., Hanson C. y Lomax. 2013. Reducing Food Loss and Waste. Creating a Sustainable Food Future. Technical report World Resources Institute. Online: https://goo.gl/XodoaL.

Llorens-Ivorra, Cristóbal, Ilona Arroyo-Ba nuls, Joan Quiles-Izquierdo y Miguel RichartMartínez. 2017. "Evaluación del equilibrio alimentario de los menús escolares de la Comunidad Valenciana (España) mediante un cuestionario." Gaceta Sanitaria . Online: https:/ / doi.org/10.1016/j.gaceta.2017.01.010.

Martins, Margarida Liz, Luís M. Cunha, Sara S.P. Rodrigues y Ada Rocha. 2014. “Determination of plate waste in primary school lunches by weighing and visual estimation methods: A validation study." Waste Management 34(8):1362-1368. Online: https://doi.org/10.1016/j.wasman.2014. 03.020 . 
Ministerio de Agricultura y Pesca, Alimentación y Medio Ambiente. 2017. Estrategia más alimento, menos desperdicio 2017 - 2020. Catálogo de Publicaciones de la AGE. Online: https://goo.gl/ SgWqqv.

Papargyropoulou, Effie, Rodrigo Lozano, Julia K. Steinberger, Nigel Wright y Zaini bin Ujang. 2014. "The food waste hierarchy as a framework for the management of food surplus and food waste." Journal of Cleaner Production 76:106-115. Online: https://doi.org/10.1016/j.jclepro.2014.04.020.

Parfitt, Julian, Mark Barthel y Sarah Macnaughton. 2010. "Food waste within food supply chains: quantification and potential for change to 2050." Philosophical Transactions of the Royal Society of London B: Biological Sciences 365(1554):3065-3081. Online: https://goo.gl/JJuYsJ.

Priefer, Carmen, Juliane Jörissen y Klaus-Rainer Bräutigam. 2016. "Food waste prevention in Europe - A cause-driven approach to identify the most relevant leverage points for action." Resources, Conservation and Recycling 109:155-165. Online: https://doi.org/10.1016/j.resconrec.2016.03. 004 .

Quested, T.E., E. Marsh, D. Stunell y A.D. Parry. 2013. "Spaghetti soup: The complex world of food waste behaviours." Resources, Conservation and Recycling 79:43 - 51. Online: https:/ /doi.org/ 10.1016/j.resconrec.2013.04.011.
Rodriguez-Tadeo, A., Patiño Villeno B. Periago Caston M. J. Ros Berruezo G. \& González MartínezLacuesta E. 2014. "Evaluando la aceptación de alimentos en escolares; registro visual cualitativo frente a análisis de residuos de alimentos." $\mathrm{Nu}$ trición Hospitalaria 29:1054-1061. Online: https: //goo.gl/LqXbp7.

Secondi, Luca, Ludovica Principato y Tiziana Laureti. 2015. "Household food waste behaviour in EU-27 countries: A multilevel analysis." Food Policy 56:25-40. Online: https://doi.org/10.1016/j. foodpol.2015.07.007.

Stenmarck, Âsa, Carl Jensen, Tom Quested y Graham Moates. 2016. Estimates of European food waste levels. Technical report European Commission (FP7), Coordination and Support Action CSA. Online: https://goo.gl/dLF7Bo.

Thyberg, Krista L. y David J. Tonjes. 2016. “Drivers of food waste and their implications for sustainable policy development." Resources, Conservation and Recycling 106:110-123. Online: https: //doi.org/10.1016/j.resconrec.2015.11.016.

Wilkie, Ann C., Ryan E. Graunke y Camilo Cornejo. 2015. "Food Waste Auditing at Three Florida Schools." Sustainability 7(2):1370-1387. Online: https://goo.gl/qUJQbn.

World Resources Institute. 2016. Food Loss and Waste Accounting and Reporting Standard. In Food Loss + Waste. Online: https://goo.gl/ ERFupF. 